\title{
Shared responsibility for treatment-related morbidity for prostate cancer
}

\author{
Joseph L. Chin, MD, FRCSC; ${ }^{*}$ Glenn Bauman, MD, FRCPC ${ }^{\dagger}$
}

See related article on page 105.

Can Urol Assoc J 2010;4(2):112-3

T reatment of locally advanced or locally recurrent prostate cancer often involves both radical prostatectomy and external beam radiotherapy; a multidisciplinary management approach is encouraged. Various researchers have published on disease eradication or control, progression-free survival and overall survival. In the adjuvant setting, there is Level 1 evidence now from three randomized trials, and supportive evidence from a multitude of non-randomized case series. The evidence shows therapeutic benefit to radiotherapy following radical prostatectomy in high-risk patients. ${ }^{1-3}$ In the salvage setting, there is also retrospective evidence, albeit lower-level, from individual centres, as well as pooled data showing good results in appropriate patients. ${ }^{4}$

Urologists are obviously very cognizant of morbidities related to radical prostatectomy. Potential surgical candidates are counselled on the risk of postoperative urinary incontinence, erectile dysfunction, bladder neck contractures and strictures, perioperative concerns and rare intra-operative injuries. There have been widespread efforts in the urologic community to make radical prostatectomy a safe, "routine" procedure, and to further improve on the morbidity profile with meticulous "anatomic" dissection, nerve-sparing techniques, anti-incontinence procedures, postoperative bladder re-training and penile rehabilitation. ${ }^{5-7}$

Postoperatively, if the patient is a candidate for adjuvant or salvage radiotherapy, referral is made to our radiation oncology colleagues. Urologists may acknowledge and often regard post-prostatectomy radiotherapy-related complications as generally well-tolerated and transient, often deferring such discussions to our radiation oncology colleagues. For instance, in the urologic literature there is a randomized study on 100 patients with adjuvant radiotherapy (60 Gy) or surveillance after radical prostatectomy reporting no difference at 24 months in those achieving complete continence (77 vs. $83 \%) .{ }^{8}$ The Southwest Oncology Group (SWOG) 8794/ National Cancer Institute of Canada (NCIC) PR-2 study involving 431 pT3 patients randomized to either early adjuvant radiotherapy or delayed treatment at relapse reported "well tolerated" short-term toxicity. At 5 years, "statistically insignificant" late toxicity included tenesmus $11 \%$ versus $19 \%(p=0.16), 18 \%$ versus $23 \%$ for urinary frequency $(p=0.55)$ and $78 \%$ versus $88 \%$ for erectile dysfunction $(p=0.14)$. However, other specific adverse effects were more common with radiotherapy, including urethral strictures $(17.8 \%$ vs. $9.5 \%)$ and total urinary incontinence $(6.5 \%$ vs. $2.8 \%){ }^{1}$ Thus, the true bother and negative effects on quality of life with sequential therapy may be under-appreciated by urologists.

Radiation oncologists have been equally (if not more) diligent in searching ways to minimize radiation-related toxicities. Technical advances, such as computed tomography simulation and computerized beam shaping devices (multileaf collimators), have enabled more sophisticated "dose painting" using three-dimensional conformal or intensity modulated radiotherapy techniques. Better imaging and pathology correlates with patterns of recurrence post-prostatectomy have led to consensus recommendations for adjuvant and salvage treatment volumes. ${ }^{9}$ In addition, refinement in patient selection and timing of treatment (i.e., delaying start of radiotherapy until continence has returned postprostatectomy), and pharmacologic advances have all contributed to improving the therapeutic index. ${ }^{10}$ In the salvage setting, an example of ongoing efforts to decrease morbidity is a prospective study of intensity modulation compared to three-dimensional conformal radiotherapy with promising early results in terms of acute gastrointestinal and genitourinary toxicity. ${ }^{11}$ These improvements may be expected to lower toxicity rates compared to those reported in the literature (which generally reflect, older, non-conformal treatment techniques). However, in spite of the improved toxicity profile from these technical improvements, urologists may still need to attend to troublesome radiation-induced hematuria, exacerbation of urethral strictures $(10 \%)$, as well as erectile dysfunction (up to $80 \%$ to $90 \%$ ) that can occur with combined treatment. Likewise, colorectal surgeons and endoscopists may need to deal with persistent rectal bleeding problems, although this toxicity may be less problematic given the somewhat lower doses used in the adjuvant/salvage setting compared to the primary radiotherapy setting. 
Thus, in spite of proven benefit of sequential multidisciplinary treatment for many locally advanced and locally recurrent prostate cancers, and parallel efforts by urologists and radiation oncologists to improve patient acceptance for their respective treatment, our perspectives on treatmentrelated toxicities tend to be somewhat specialty-specific and "siloed" and we under-appreciate the combined toxicities experienced by our patients. Accordingly, our counselling to patients may be inadequate. The retrospective study by Sia and colleagues highlights symptom-related bother and toxicities from postoperative radiotherapy for prostate cancer. ${ }^{12}$ Urologists and radiation oncologists need to share the responsibility for pre-treatment counselling, amelioration and management of the combined, sometimes compounded, treatment-related toxicities for our patients in a collaborative manner. Both specialties also have a responsibility for promoting and encouraging enrollment of patients in trials of adjuvant and salvage radiotherapy (i.e., PR13/Radicals; RTOG [Radiation Therapy Oncology Group] 0534/SPPORT [Short-term androgen deprivation with pelvic lymph node or prostate bed only radiotherapy]) that will help delineate the cost-benefit ratio of combined modality therapy for patients treated with the benefit of the newer surgical and radiotherapeutic technologies.

From the ${ }^{*}$ Division of Urology, London Health Sciences Centre, London, ON; ${ }^{\dagger}$ Chair, Department of Oncology, University of Western Ontario, London Health Sciences Centre, London, ON

Competing interests: None declared.

This paper has been peer-reviewed.

\section{References}

1. Thompson I, Tangen C, Paradelo J, et al. Adjuvant radiotherapy for pathological T3NOMO prostate cancer significantly reduces metastases and improves survival: Long term followup of a randomized trial. J Urol 2009; 181:956-62.

2. Bolla M, van Poppel H, Collette L, et al. European Organization for Research and Treatment of Cancer. Postoperative radiotherapy after radical prostatectomy: a randomised controlled trial (EORTC trial 22911). Lancet 2005;366:572-8.

3. Wiegel T, Bottke D, Steiner U, et al. Phase III postoperative adjuvant radiotherapy after radical prostatectomy compared with radical prostatectomy alone in pT3 prostate cancer with postoperative undetectable prostate-specific antigen: ARO 96-02/AUO AP 09/95. J Clin Ocol 2009;27:2924-30.

4. Stephenson A, Scardino P, Kattan M, et al. Predicting the outcome of salvage radiation therapy for recurrent prostate cancer after radical prostatectomy. J Clin Oncol 2007;25:2035-41.

5. Walsh PC. The discovery of the cavernous nerves and development of nerve sparing radical retropubic prostatectomy. J Urol 2007;177:1632-5.

6. Walsh PC, Marschke P, Ricker D, et al. Patient-reported urinary continence and sexual function after anatomic radical prostatectomy. Urology 2000;55:58-61.

7. Müller $A$, Parker $M$, Waters $B W$, et al. Penile rehabilitation following radical prostatectomy: predicting success. J Sex Med 2009;6:2806-12.

8. Van Cangh PJ, Richard F, Lorge F, et al. Adjuvant radiation therapy does not cause urinary incontinence after radical prostatectomy: results of a prospective randomized study. J Urol 1998;159:164-6.

9. Michalski JM, Lawton C, El Naqa I, et al. Development of RTOG consensus guidelines for the definition of the clinical target volume for postoperative conformal radiation therapy for prostate cancer. Int J Radiat Oncol Biol Phys 2010;76:361-8.

10. Roach $M$ 3rd. Reducing the toxicity associated with the use of radiotherapy in men with localized prostate cancer. Urol Clin North Am 2004;31:353-66.

11. Alongi F, Fiorino C, Cozzarini C, et al. IMRT significantly reduces acute toxicity of whole-pelvis irradiation in patients treated with post-operative adjuvant or salvage radiotherapy after radical prostatectomy. Radiother Oncol 2009;93:207-12.

12. Sia $M$, Rodrigues $G$, Menard $C$, et al. Treatment-related toxicity and symptom-related bother following postoperative radiotherapy for prostate cancer. Can Urol Assoc J 2010;4:105-11.

Correspondence: Dr. Joseph L. Chin, Division of Urology, London Health Sciences Centre, University of Western Ontario, 800 Commissioners Rd. E., London 0N N6A 4G5; ichin@hhsc.on.ca 